\title{
Subcycle Control of Electron-Electron Correlation in Double Ionization
}

\author{
Li Zhang, ${ }^{1}$ Xinhua Xie, ${ }^{1}$ Stefan Roither, ${ }^{1}$ Yueming Zhou, ${ }^{2,3}$ Peixiang Lu, ${ }^{2,3,}{ }^{*}$ Daniil Kartashov, ${ }^{1}$ Markus Schöffler, \\ Dror Shafir, ${ }^{5}$ Paul B. Corkum, ${ }^{4}$ Andrius Baltuška, ${ }^{1}$ André Staudte, ${ }^{4}$ and Markus Kitzler ${ }^{1, \dagger}$ \\ ${ }^{1}$ Photonics Institute, Vienna University of Technology, A-1040 Vienna, Austria \\ ${ }^{2}$ School of Physics, Huazhong University of Science and Technology, and \\ Wuhan National Laboratory for Optoelectronics, Wuhan 430074, China \\ ${ }^{3}$ Key Laboratory of Fundamental Physical Quantities Measurement of Ministry of Education, Wuhan 430074, China \\ ${ }^{4}$ Joint Laboratory for Attosecond Science of the National Research Council and the University of Ottawa, \\ Ottawa, Ontario, Canada K1A OR6 \\ ${ }^{5}$ Department of Physics of Complex Systems, Weizmann Institute of Science, Rehovot 76100, Israel \\ (Received 9 April 2013; revised manuscript received 26 February 2014; published 14 May 2014)
}

\begin{abstract}
Double ionization of neon with orthogonally polarized two-color (OTC) laser fields is investigated using coincidence momentum imaging. We show that the two-electron emission dynamics in nonsequential double ionization can be controlled by tuning the subcycle shape of the electric field of the OTC pulses. We demonstrate experimentally switching from correlated to anticorrelated two-electron emission, and control over the directionality of the two-electron emission. Simulations based on a semiclassical trajectory model qualitatively explain the experimental results by a subcycle dependence of the electron recollision time on the OTC field shape.
\end{abstract}

DOI: 10.1103/PhysRevLett.112.193002

PACS numbers: $33.20 . \mathrm{Xx}, 32.80 . \mathrm{Rm}$

Angström and attosecond control of free electron wave packets is one of the pinnacles of attosecond science. Orthogonally polarized two-color (OTC) laser fields allow us to control the motion of field-ionizing electronic wave packets both in time and space $[1,2]$. In OTC pulses time and space are connected and thus an attosecond time scale is established in the polarization plane for both the emitted and the recolliding wave packets $[3,4]$. OTC pulses have been proposed to increase the efficiency [5] and to allow control over the polarization state of high-harmonic radiation [6], and they have been used to interrogate atomic and molecular orbital structure [7-9] via high harmonic radiation. The ability to steer electrons with two-color laser fields has led to proposals for using them in laser induced electron diffraction [10] and double ionization [11,12].

Here, we report on experiments and semiclassical simulations of nonsequential double ionization (NSDI) in orthogonally polarized 800 and $400 \mathrm{~nm}$ laser fields. We show that these OTC fields provide control over the emission dynamics of two electrons from neon atoms in the intensity regime of NSDI. By manipulating the subcycle shape of the OTC field we demonstrate switching from correlated to anticorrelated two-electron emission along the polarization direction of the fundamental field. Simultaneously, the OTC pulses provide control over the two-electron emission direction along the second-harmonic field axis, similar to a single color carrier-envelope phase stabilized few cycle pulse [13]. Finally, we find that the NSDI rate in OTC pulses is very sensitive to the initial transverse momentum of the recolliding electron.

In our experiments the OTC pulses were produced by combining an $800 \mathrm{~nm}$ laser pulse, frequency $\omega$, and its second harmonic pulse, frequency $2 \omega$, polarized along $x$ and $z$, respectively, in a collinear geometry at a rate of $5 \mathrm{kHz}$. The laser peak intensity in either color was $I_{800 \mathrm{~nm}}=I_{400 \mathrm{~nm}}=$ $(2 \pm 0.2) \times 10^{14} \mathrm{~W} / \mathrm{cm}^{2}$. The durations (FWHM) of the fundamental (46 fs) and second harmonic pulse (48 fs) were measured by using second-harmonic frequency resolved optical gating (FROG) and self-diffraction FROG, respectively. Temporal overlap of the two pulses was ensured by compensating for their different group velocities with calcite plates and a pair of fused silica wedges. The electric field of the OTC pulses can be written as (atomic units are used unless otherwise stated) $\vec{E}(t)=$ $f_{x}(t) \cos (\omega t) \vec{e}_{x}+f_{z}(t) \cos (2 \omega \mathrm{t}+\Delta \varphi) \vec{e}_{z}$, with $\Delta \varphi$ the relative phase of the two colors. Variations of $\Delta \varphi$ by fine steps of one of the wedges allows us to control the waveform of the OTC pulse on a subcycle time scale $[1,3]$. The threedimensional momentum vector of electrons and ions emitted from neon atoms upon interaction with the OTC pulses was measured as a function of $\Delta \varphi$ using cold-target recoil-ion momentum spectroscopy (COLTRIMS) [14]. The relative phase was calibrated by the peaks of the $\mathrm{Ne}^{+}$yield modulation measured at lower intensities $[15,16]$. The COLTRIMS setup was described in detail previously [15]. In short, electrons and ions created in the laser focus were guided by weak uniform electric $(1.8 \mathrm{~V} / \mathrm{cm})$ and magnetic $(10.5 \mathrm{G})$ fields onto two multihit position- and time-sensitive detectors with delay line anodes for position readout. The ion rate was adjusted to $\approx 0.3$ per laser shot. From the measured time of flight and position of each particle, its three dimensional momentum vector was calculated.

The process of NSDI, i.e., the emission of two electrons from an atom or molecule due to inelastic scattering of an 
electron that is driven back to its parent ion by a strong laser field [17-19], has become a test bed for the investigation $[13,20]$ and theoretical description $[21,22]$ of the dynamics of electron correlation on few to sub-fs time scales. The measured yield of $\mathrm{Ne}^{2+}$ and $\mathrm{Ne}^{+}$ions as a function of $\Delta \varphi$ is shown in Fig. 1(a). At the peak intensities used in the experiments, the $\mathrm{Ne}^{+}$yield is close to saturation and becomes independent of $\Delta \varphi$. However, the measured yield of $\mathrm{Ne}^{2+}$ strongly depends on $\Delta \varphi$ with maxima around $\Delta \varphi \approx(n+0.5) \pi, n \in \mathbb{N}$. In contrast, tunneling theory [23] for two independent tunneling steps predicts that the $\mathrm{Ne}^{2+}$ yield peaks at $\Delta \varphi=n \pi$ [see Fig. 1(a)].

In order to gain insight into the experimentally observed two-electron emission dynamics, we performed simulations using a semiclassical model [24]. In this model, the first electron is assumed to be tunnel ionized into the continuum with a probability according to tunneling theory [23]. The electron is placed at the tunneling exit with zero longitudinal momentum and a Gaussian distribution of transverse momentum [25]. The initial condition of the second (bound) electron is a microcanonical distribution in the screened potential of the doubly charged ion with an energy of -1.5 a.u. (the second ionization potential of $\mathrm{Ne}$ ). Thereafter, the evolution of the tunneling and bound electron is determined by the coupled classical equations of motion

$$
\frac{d^{2} \vec{r}_{i}}{d t^{2}}=-\vec{E}(t)-\vec{\nabla}\left[V_{n e}\left(\vec{r}_{i}\right)+V_{e e}\left(\vec{r}_{1}, \vec{r}_{2}\right)\right], \quad i=(1,2),
$$
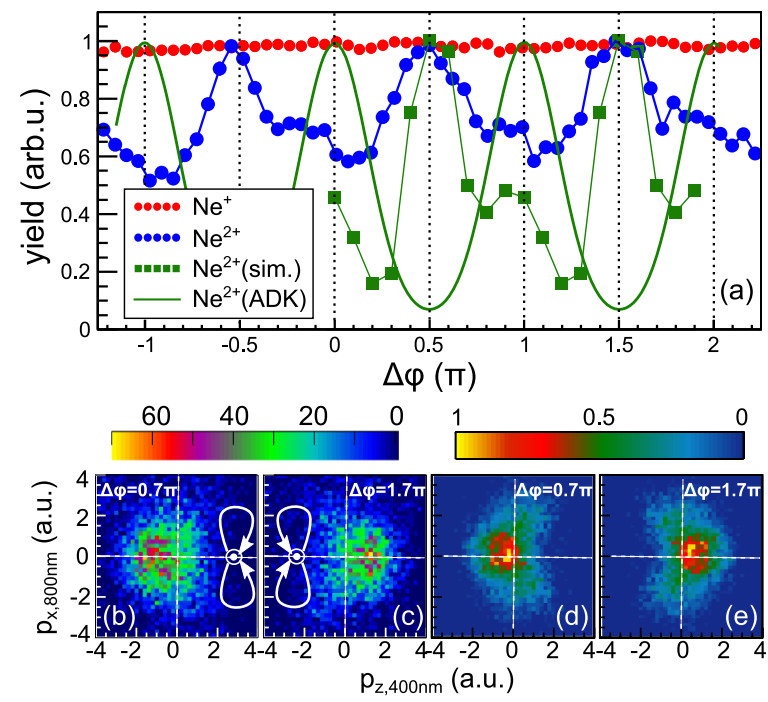

FIG. 1 (color online). (a) Measured normalized yield of $\mathrm{Ne}^{+}$ (red dots) and $\mathrm{Ne}^{2+}$ (blue dots) ions over relative phase $\Delta \varphi$. The solid green line represents the calculated normalized $\mathrm{Ne}^{2+}$ yield assuming two independent tunneling steps. Green squares represent the results from the semiclassical trajectory model (see text). (b),(c) Measured momentum distributions of $\mathrm{Ne}^{2+}$ in the polarization plane of the OTC field for $\Delta \varphi=0.7 \pi$ (b) and $\Delta \varphi=1.7 \pi$ (c). (d),(e) Same as (b),(c) but simulated using the trajectory model. with $\vec{E}(t)$ the OTC laser field as defined above with an envelope that has a constant amplitude for the first 6 cycles and then linearly turns off during 2 cycles of the $800 \mathrm{~nm}$ field. $V_{n e}\left(\vec{r}_{i}\right)=-2 / \sqrt{r_{i}^{2}+a^{2}}$ is the ionic potential with a soft-core parameter $a=0.5$ to avoid the Coulomb singularity, and $V_{e e}\left(\vec{r}_{1}, \vec{r}_{2}\right)=1 /\left|\vec{r}_{1}-\vec{r}_{2}\right|$ is the electron-electron interaction potential. For simplicity, we restricted the motion of the electrons to the laser polarization plane. This restriction underestimates the diffusion of the electron wave packet and overestimates recollision ionization during multiple returns. To account for that we have excluded double ionization (DI) events occurring at later returns. The dependence of the double ionization yield on $\Delta \varphi$ predicted by the model is overlaid on the experimental results in Fig. 1(a). The experimental data, in particular, the maxima or minima and the small knee structures in between them, are reproduced very well. The discrepancy in the modulation depth of the yield may be caused by intensity averaging in the laser focus not taken into account in the calculations, by a jitter of $\Delta \varphi$ in the experiments, or by quantum effects that are not captured by the semiclassical model.

In NSDI the two electrons can be ejected either via a correlated scenario, where both electrons are emitted into the same hemisphere, or via an anticorrelated scenario, where both electrons are preferentially emitted into opposite hemispheres. Insight into the two-electron emission dynamics can be gained from correlated two-electron momentum spectra. This requires the detection of at least one electron in addition to the doubly charged ion. The momentum vector of the second electron can then be calculated from the relation $\vec{p}_{1}+\vec{p}_{2}=-\vec{p}^{\text {ion }}$. In our experiment the statistics is too small to plot the correlated two-electron spectra, therefore, we will restrict the analysis to $\vec{p}^{\text {ion }}$. Analysis of the spectra of the sum momentum vector in terms of their mean values and widths along $p_{x}$ and $p_{z}$, however, allows obtaining detailed insight into the correlated electron emission dynamics, as we will show in the following.

Figures 1(b) and 1(c) show measured $\mathrm{Ne}^{2+}$ momentum distributions in the polarization plane for two values of $\Delta \varphi$. The distributions are symmetric about the $\omega$-field axis $(x)$, while they show a pronounced asymmetry that depends on $\Delta \varphi$ along the $2 \omega$-field axis (z). The simulations nicely reproduce the measured spectra [Figs. 1(d) and 1(e)]. This symmetry dependence reflects the spatial asymmetry in the OTC field, where the photoelectron returns to the ion under a time-dependent angle $\pm \alpha$ with the $2 \omega$-field axis $[3,4,7]$ [see insets in Figs. 1(b) and 1(c)]. To study the effects of the varying spatial asymmetry with $\Delta \varphi$ on the two-electron emission, we show in Figs. 2(b) and 2(c) the momentum spectra of $\mathrm{Ne}^{2+}$ for the $p_{x}(800 \mathrm{~nm})$ and $p_{z}(400 \mathrm{~nm})$ momentum components separately. It can be seen by the $2 \pi$ periodicity of the $p_{z, 400 \mathrm{~nm}}$ asymmetry [Fig. 2(b)] that both electrons are periodically emitted into the same hemisphere along the $400 \mathrm{~nm}$ field direction, while no asymmetry is 


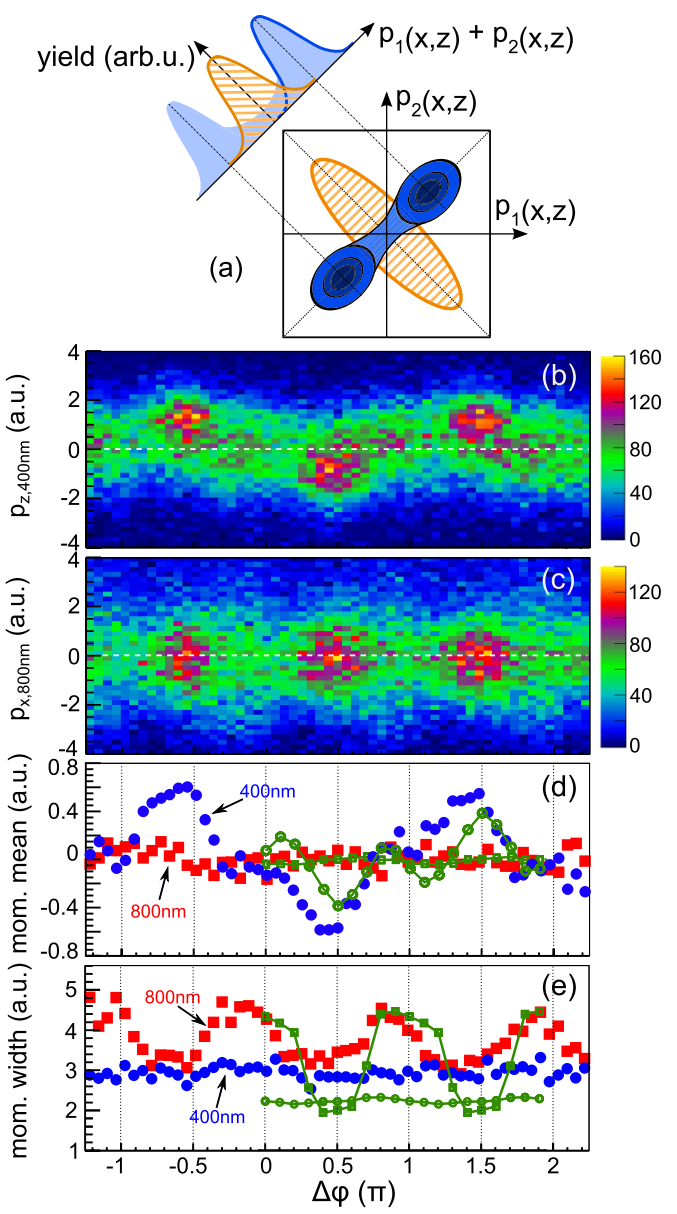

FIG. 2 (color online). (a) Signatures of correlated (blue, full) and anticorrelated (orange, hatched) two-electron emission dynamics in two-electron (lower right) and ion (upper left) momentum spectra. (b),(c) $\mathrm{Ne}^{2+}$ momentum along $p_{z}$ (b) and $p_{x}$ (c) with the other directions integrated over as a function of relative phase $\Delta \varphi$. (d) $\Delta \varphi$-dependent mean value of $p_{z}$ (filled blue dots) and $p_{x}$ (filled red squares). (e) Width of $p_{z}$ (filled blue dots) and $p_{x}$ (filled red squares). The corresponding simulated results in (d) and (e) are shown by open green circles and squares, respectively.

found along the $800 \mathrm{~nm}$ field axis [Fig. 2(c)]. For obtaining detailed information about the electron-electron correlation we now analyze the $\Delta \varphi$ dependence of the $\mathrm{Ne}^{2+}$ momentum distributions along $p_{z}$ and $p_{x}$ in terms of their mean momentum value and spectral width (FWHM).

Figure 2(a) illustrates the information contained in the spectral mean value and width of the ion momentum distribution $p_{(x, z)}^{\text {ion }}=-\left(p_{1(x, z)}+p_{2,(x, z)}\right)$. Correlated twoelectron emission (indicated in blue) results either in a large width and zero mean value, if it takes place symmetrically into both hemispheres (full blue area), or in a narrow width and large mean value if it happens dominantly into one hemisphere (blue thick line). Anticorrelated two-electron emission (indicated in hatched orange) manifests itself as a narrow ion momentum spectrum with zero mean value.
Figures 2(d) and 2(e) show the mean value and width of the $\mathrm{Ne}^{2+}$ momentum along $p_{x, 800 \mathrm{~nm}}$ and $p_{z, 400 \mathrm{~nm}}$ as red squares and blue circles, respectively. While the width along the $400 \mathrm{~nm}$ direction is constant with $\Delta \varphi$, the mean value strongly modulates with the relative phase. This is consistent with a correlated two-electron emission [as sketched by the blue thick line in the ion momentum spectrum in Fig. 2(a)], where both electrons are ejected dominantly into the same hemisphere with $p_{z}>0$ for $\Delta \varphi \approx$ $0.4 \pi+n 2 \pi$ and to $p_{z}<0$ for $\Delta \varphi \approx 1.4 \pi+n 2 \pi, n \in \mathbb{N}$; very close to those relative phases where the total double ionization probability maximizes [cf. Fig. 1(a)]. Thus, along the $400 \mathrm{~nm}$ direction the OTC pulse has a comparable effect on the photoelectrons as a carrier-envelope phase stabilized few-cycle laser pulse [13]. This reflects the characteristic of OTC fields where trajectories from successive half-cycles of the $800 \mathrm{~nm}$ field are recolliding under the same angle relative to the $400 \mathrm{~nm}$ field [3,7] [cf. insets in Figs. 1(b) and 1(d)].

In contrast to the $400 \mathrm{~nm}$ direction, the mean value of the $p_{x, 800 \mathrm{~nm}}$ momentum component of $\mathrm{Ne}^{2+}$ stays roughly constant over $\Delta \varphi$ [Fig. 2(d)]. The $p_{x, 800 \mathrm{~nm}}$ width, however, oscillates between 3 and 5 a.u. [Fig. 2(e)], and minimizes when the total double ionization probability maximizes [compare with Fig. 1(a)]. One scenario that can explain both the small momentum width and the increased twoelectron ionization probability at these $\Delta \varphi$, is that the two electrons are emitted into opposite $p_{x, 800 \mathrm{~nm}}$ hemispheres in a strongly anticorrelated emission scenario [as sketched by the orange spectrum in Fig. 2(a)]. For those phases where the spectral width is large, the data imply that the emission happens in a correlated manner with emissions alternately into both hemispheres $p_{x}>0$ and $p_{x}<0$ which leads to spectra as sketched by the blue areas in Fig. 2(a). Thus, our measurements demonstrate that by using OTC laser fields it is possible to control the electron-electron (anti-)correlation during NSDI by using $\Delta \varphi$ as the control parameter.

We will now use the semiclassical model described above to corroborate the conclusions drawn from the experiments. Figures 3(a)-3(d) show simulated two-electron momentum spectra along $p_{x, 800 \mathrm{~nm}}$ and $p_{z, 400 \mathrm{~nm}}$ for two relative phases, for which the simulated DI yield shows a maximum $(\Delta \varphi=0.6 \pi)$ and a minimum $(\Delta \varphi=0.2 \pi)$, respectively. It can be clearly seen that at $\Delta \varphi=0.2 \pi$ the two-electron emission along the $800 \mathrm{~nm}$ polarization direction is primarily correlated into the same hemisphere (quadrants 1 and 3). In contrast, at $\Delta \varphi=0.6 \pi$ the two electrons are strongly anticorrelated and emitted into opposite hemispheres (quadrants 2 and 4). Thus, the model confirms the alternation between correlated and anticorrelated emission along the $800 \mathrm{~nm}$ direction observed in the experiment. Likewise, the narrow two-electron momentum distribution with alternating asymmetry, observed along the $400 \mathrm{~nm}$ direction in the experiment, is confirmed by the simulations [Figs. 3(b) and 3(d)]. Simulated results of the 


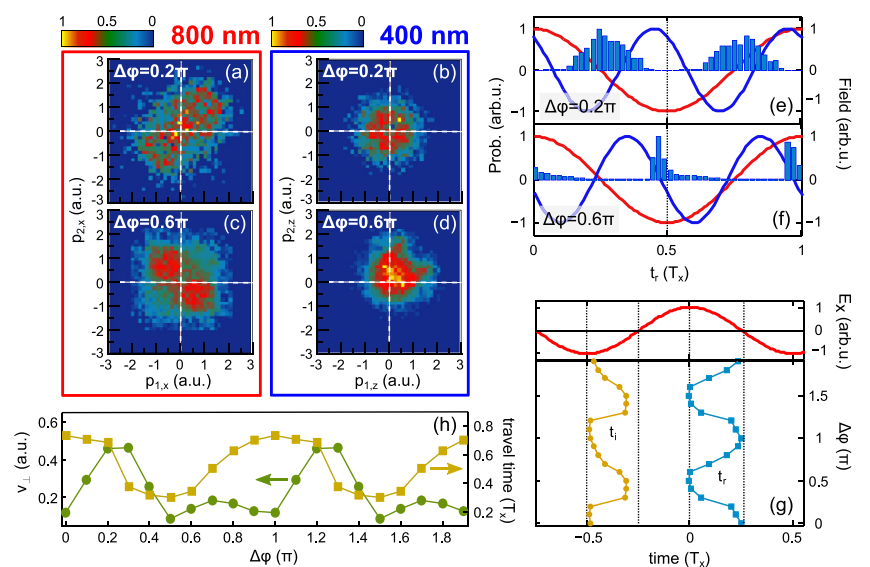

FIG. 3 (color online). (a),(b) Simulated two-electron momentum distributions along the $800 \mathrm{~nm}$ (a) and $400 \mathrm{~nm}$ (b) polarization directions for $\Delta \varphi=0.2 \pi$. (b),(d) Same as (a) and (c) but for $\Delta \varphi=0.6 \pi$. (e),(f) DI yield versus laser phase at the instant of recollision for $\Delta \varphi=0.2 \pi$ (e) and $\Delta \varphi=0.6 \pi$ (f). $T_{x}$ denotes the optical period of the $800 \mathrm{~nm}$ laser field. (g) Mean values of the time of recollision (blue squares) and the tunnel ionization time of the first electron (orange circles) as a function of $\Delta \varphi$. The top panel shows a cycle of the $800 \mathrm{~nm}$ laser field. (h) Mean value of the initial transverse velocity at the instant of tunneling (green circles, left axis) and the mean value of the travel time (orange squares, right axis) over $\Delta \varphi$ for those trajectories that eventually lead to DI.

width and mean value along $p_{x, 800 \mathrm{~nm}}$ and $p_{z, 400 \mathrm{~nm}}$ for a variation of $\Delta \varphi$ over a full period of $2 \pi$ are shown in Figs. 2(d) and 2(e) by the green symbols, and good agreement with the experimental values is achieved.

For further insight into the origin of the $\Delta \varphi$-dependent electron-electron correlations we traced the evolution of those semiclassical trajectories that lead to DI. In Figs. 3(e) and 3(f) we show the probability distribution of the time of recollision $t_{r}$ (blue bars) for $\Delta \varphi=0.2 \pi$ and $\Delta \varphi=0.6 \pi$, respectively. Here, $t_{r}$ is defined as the instant of the closest approach of the tunnel ionized electron to the parent ion. It can be seen that for $\Delta \varphi=0.2 \pi$ recollision occurs predominantly around the zero crossing of the $800 \mathrm{~nm}$ field (just after the field maximum of the $400 \mathrm{~nm}$ field). If both electrons ionize immediately after the recollision, the classically expected momentum values that the two electrons gain from the laser field are given by [26] $p_{x, 800 \mathrm{~nm}}=-A_{x}\left(t_{r}\right)$ and $p_{z, 400 \mathrm{~nm}}=-A_{z}\left(t_{r}\right)$, with $A_{x, z}(t)=-\int_{-\infty}^{t} E_{x, z}\left(t^{\prime}\right) \mathrm{d} t^{\prime}$ the vector potential along the $x$ and $z$ direction, respectively. Thus, when the two electrons are released around $E_{x}(t) \approx 0$ and around a maximum of $E_{z}(t)$, they gain the same nonzero momentum along the $p_{x, 800 \mathrm{~nm}}$ direction and nearly zero momentum along the $p_{z, 400 \mathrm{~nm}}$ direction, resulting in the correlated distributions shown in Figs. 3(a) and 3(b). At $\Delta \varphi=0.6 \pi$, on the other hand, recollision occurs predominantly just before the maximum of the $800 \mathrm{~nm}$ field and around the zero crossing of the $400 \mathrm{~nm}$ field [Fig. 3(f)]. Consequently, the two electrons gain nonzero momentum along the $p_{z, 400 \mathrm{~nm}}$ direction, in agreement with the asymmetric momentum distribution in Fig. 3(d). For the strong anticorrelated back-to-back emission of the two electrons along $p_{x, 800 \mathrm{~nm}}$ that we observe both in experiment and simulation [Fig. 3(c)], the two electrons must gain relatively large comparable momenta into opposite directions. Such dynamics cannot be explained simply by the timing of the recollision, but may result from different initial velocities at the ionization event after the recollision [27] due to electron-electron Coulomb interaction, and to a certain extent also from a subcycle time difference between the ionization events of the two electrons [20].

We conclude that the simulations reveal a correspondence between the emission dynamics of the two electrons, and the subcycle variations of the recollision time when $\Delta \varphi$ is changed. When $\Delta \varphi$ is varied over a full period of $2 \pi$, both the time of recollision $\left(t_{r}\right)$ and the time of ionization of the first electron $\left(t_{i}\right)$ periodically vary over roughly a quarter (half)-cycle of the 800 (400 nm) field, as shown in Fig. 3(g). As the recollision time gradually varies between the zero crossing and the maximum of the $800 \mathrm{~nm}$ field, the electron distributions vary between the correlated and anticorrelated behavior, which results in the oscillations of the width of the ion momentum spectra along the $p_{x, 800 \mathrm{~nm}}$ direction [Fig. 2(d)], as described above. Because recollisions occur every half cycle of the $800 \mathrm{~nm}$ field [Figs. 3(e) and 3(f)], the momentum that the two electrons gain from the field and impart to the ion upon inelastic scattering taking place during half-cycles of different polarity, averages out over the duration of the multicycle pulse. As a result, the mean value of the measured ion momentum stays zero independent of $\Delta \varphi$ [Fig. 2(d)]. In contrast, for the $400 \mathrm{~nm}$ field the periodicity of the recollisions is one laser cycle, not half a laser cycle. Thus, the momenta from two successive scattering events do not cancel. As a result, the mean value of the ion momentum oscillates when the recollision time moves with respect to the phase of the $400 \mathrm{~nm}$ field [Fig. 2(d)].

Finally, the model can also be used to qualitatively understand the modulation of the DI yield with $\Delta \varphi$ shown in Fig. 1(a). Because of the diffusion of the recolliding electron wave packet, the probability of recollision decreases when the travel time of the tunneled electron increases. Figure 3(h) shows the travel time of the tunneled electron as a function of $\Delta \varphi$. It can be seen that this quantity is smallest for $0.4 \pi \lesssim \Delta \varphi \lesssim 0.6 \pi$. In OTC fields, effective recollision also requires a proper initial transverse velocity at tunneling [1], the value of which depends on $\Delta \varphi$. Larger transverse velocity means lower tunneling probability [25] and, thus, the recollision probability is highest at those relative phases that require the smallest initial transverse velocity. The smallest mean value of the initial transverse momentum is obtained for $0.5 \pi \lesssim \Delta \varphi \lesssim \pi$ [Fig. 3(h)]. Combining the two considered effects, maximum DI yield 
is predicted around $\Delta \varphi \approx 0.5 \pi$, in very good agreement with the measured value [Fig. 1(a)].

In conclusion, we used the COLTRIMS technique to investigate for the first time atomic double ionization by OTC laser fields. We showed experimentally that by tuning the shape of the electric field of the OTC pulses on the subcycle scale it is possible to control the two-electron emission dynamics in nonsequential double ionization, and to dictate whether the two electrons are predominantly emitted in a correlated or anticorrelated manner. With the help of simulations based on a semiclassical trajectory model we are able to qualitatively explain the experimental results by subcycle changes of the recollision time when the relative phase of the two colors is varied.

We acknowledge funding by the Austrian Science Fund (FWF) under Grants No. P21463-N22, No. P25615-N27, and No. SFB-F49 NEXTlite, by a starting grant from the ERC (project $\mathrm{CyFi}$ ), and by the National Natural Science Foundation of China (Grants No. 11234004 and No. 61308030) and 973 Program of China (Grant No. 2011CB808103).

*lupeixiang@mail.hust.edu.cn

${ }^{\dagger}$ Corresponding author. markus.kitzler@tuwien.ac.at

[1] M. Kitzler and M. Lezius, Phys. Rev. Lett. 95, 253001 (2005).

[2] M. Kitzler, K. O'Keeffe, and M. Lezius, J. Mod. Opt. 53, 57 (2006).

[3] M. Kitzler, X. Xie, A. Scrinzi, and A. Baltuska, Phys. Rev. A 76, 011801 (2007).

[4] M. Kitzler, X. Xie, S. Roither, A. Scrinzi, and A. Baltuska, New J. Phys. 10, 025029 (2008).

[5] C. M. Kim, I. J. Kim, and C. H. Nam, Phys. Rev. A 72, 33817 (2005).

[6] C. Ruiz, D. J. Hoffmann, R. Torres, L. E. Chipperfield, and J. P. Marangos, New J. Phys. 11, 113045 (2009).
[7] D. Shafir, Y. Mairesse, D. M. Villeneuve, P. B. Corkum, and N. Dudovich, Nat. Phys. 5, 412 (2009).

[8] H. Niikura, N. Dudovich, D. M. Villeneuve, and P. B. Corkum, Phys. Rev. Lett. 105, 053003 (2010).

[9] H. Niikura, H. Wörner, D. M. Villeneuve, and P. B. Corkum, Phys. Rev. Lett. 107, 093004 (2011).

[10] R. Murray, C. Ruiz, J. P. Marangos, and M. Y. Ivanov, J. Phys. B 43, 135601 (2010).

[11] Y. Zhou, C. Huang, A. Tong, Q. Liao, and P. Lu, Opt. Express 19, 2301 (2011).

[12] L. Chen, Y. Zhou, C. Huang, Q. Zhang, and P. Lu, Phys. Rev. A 88, 043425 (2013).

[13] B. Bergues et al., Nat. Commun. 3, 813 (2012).

[14] R. Dörner, V. Mergel, O. Jagutzki, L. Spielberger, J. Ullrich, R. Moshammer, and H. Schmidt-Böcking, Phys. Rep. 330, 95 (2000).

[15] X. Xie et al., Phys. Rev. Lett. 108, 193004 (2012).

[16] X. Xie et al., New J. Phys. 15, 043050 (2013).

[17] T. Weber, H. Giessen, M. Weckenbrock, G. Urbasch, A. Staudte, L. Spielberger, O. Jagutzki, V. Mergel, M. Vollmer, and R. Dörner, Nature (London) 405, 658 (2000).

[18] A. Staudte et al., Phys. Rev. Lett. 99, 263002 (2007).

[19] A. Rudenko, V. L. B. de Jesus, T. Ergler, K. Zrost, B. Feuerstein, C. D. Schröter, R. Moshammer, and J. Ullrich, Phys. Rev. Lett. 99, 263003 (2007).

[20] N. Camus et al., Phys. Rev. Lett. 108, 073003 (2012).

[21] C. F. de Morisson Faria and X. Liu, J. Mod. Opt. 58, 1076 (2011).

[22] W. Becker, X. Liu, P. J. Ho, and J. H. Eberly, Rev. Mod. Phys. 84, 1011 (2012).

[23] G. L. Yudin and M. Y. Ivanov, Phys. Rev. A 64, 013409 (2001).

[24] D. F. Ye, X. Liu, and J. Liu, Phys. Rev. Lett. 101, 233003 (2008).

[25] N. B. Delone and V. P. Krainov, J. Opt. Soc. Am. B 8, 1207 (1991).

[26] M. Y. Ivanov, M. Spanner, and O. Smirnova, J. Mod. Opt. 52, 165 (2005).

[27] Y. Zhou, Q. Liao, and P. Lu, Phys. Rev. A 82, 053402 (2010). 